\title{
Flow diversion of a recurrent, iatrogenic basilar tip aneurysm in a pediatric patient: case report
}

\author{
Rahul A. Sastry, MD, Matthew J. Koch, MD, Benjamin L. Grannan, MD, \\ Christopher J. Stapleton, MD, William E. Butler, MD, and Aman B. Patel, MD \\ Department of Neurosurgery, Massachusetts General Hospital, Harvard Medical School, Boston, Massachusetts
}

\begin{abstract}
Endoscopic third ventriculostomy (ETV) is a common treatment for noncommunicating hydrocephalus. Although rare, vascular injury and traumatic pseudoaneurysm development during ETV have been reported. The authors present the case of a 13-year-old boy who underwent repeat ETV (rETV) for shunt and ETV failure, and who suffered an intraoperative subarachnoid hemorrhage due to iatrogenic injury to the basilar tip, with subsequent development of a pseudoaneurysm. Despite initial primary coil embolization, the aneurysm recurred and was definitively treated with flow diversion. In this report, the authors review complication rates associated with ETV and rETV as well as the emerging use of flow diversion and its applications in vessel reconstruction within the pediatric population.
\end{abstract}

https://thejns.org/doi/abs/10.3171/2017.7.PEDS17235

KEY WORDS endoscopic third ventriculostomy; basilar tip pseudoaneurysm; vascular disorders; hydrocephalus

$\mathrm{E}$ NDOSCOPIC third ventriculostomy (ETV) is a common treatment strategy for noncommunicating hydrocephalus. Although rare, vascular injury and traumatic pseudoaneurysm development during ETV has been reported. We present the case of a 13-year-old boy who underwent repeat ETV (rETV) for shunt and ETV failure, and who suffered an intraoperative subarachnoid hemorrhage (SAH) due to iatrogenic injury to the basilar tip, with subsequent development of a pseudoaneurysm.

\section{Case Report}

History and Examination

A 13-year-old boy who had undergone an ETV and ventriculoperitoneal shunting for hydrocephalus secondary to intraventricular hemorrhage (IVH) of prematurity presented to our emergency department with persistent headaches. Magnetic resonance imaging of the brain demonstrated enlarged lateral and third ventricles and closure of the prior ETV fenestration site, consistent with shunt and ETV failure. Preoperative thin-cut T2-weighted constructive interference in steady state (CISS) MRI demonstrated navigability through the right lateral ventricle, through the floor of the third ventricle, into the prepontine cistern, without evidence of frank arachnoid adhesions to the basilar artery (BA) (Fig. 1).

\section{Operation}

Given the patient's age, treatment of hydrocephalus with rETV was chosen given its potential long-term durability and lower potential complication rate. Under stereotactic guidance, a rigid endoscope was advanced into the right lateral ventricle, through the foramen of Monro, and into the third ventricle. The prior ETV fenestration site was identified as a translucent membrane between the mammillary bodies and median eminence, and was refenestrated with forceps until CSF flow was observed through the fenestration. As the balloon catheter was being prepared for introduction, arterial bleeding was observed from the fenestration site. The origin of the bleeding could not be visualized. The site was irrigated for 30 minutes until hemostasis was achieved; however, because of the amount of bleeding encountered, an external ventricular drain was placed, and the procedure was terminated.

A CT angiographic study conducted within the intraoperative CT suite demonstrated a 2-mm basilar tip pseudo-

ABBREVIATIONS BA = basilar artery; CISS = constructive interference in steady state; ETV = endoscopic third ventriculostomy; IVH = intraventricular hemorrhage; PCA = posterior cerebral artery; $\mathrm{PCOA}=$ posterior communicating artery; rETV = repeat ETV; SAH = subarachnoid hemorrhage.

SUBMITTED May 4, 2017. ACCEPTED July 17, 2017.

INCLUDE WHEN CITING Published online October 13, 2017; DOI: 10.3171/2017.7.PEDS17235. 


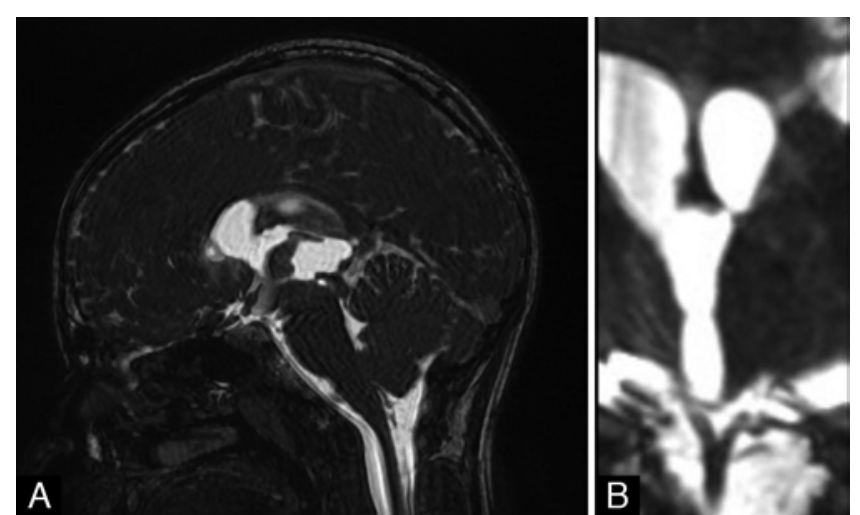

FIG. 1. Sagittal (A) and coronal (B) slices of preoperative T2-weighted CISS MRI demonstrating navigability through the right lateral ventricle and through the floor of the third ventricle into the prepontine cistern.

aneurysm, SAH, and IVH (Fig. 2A). The patient was emergently brought to the endovascular suite, where a diagnostic angiogram redemonstrated the $2 \times 2.2-\mathrm{mm}$ basilar tip aneurysm (Fig. 2B). Embolization was then performed with 3 Penumbra SMART coils placed within the dome of the aneurysm, with satisfactory modified RaymondRoy Class IIIb closure (Fig. 3). The patient awoke without neurological deficit and was monitored for vasospasm. His shunt was reinternalized on postoperative Day 14.

\section{Postoperative Course}

Follow-up angiography conducted on postoperative Day 17 demonstrated aneurysm regrowth to a size of $2.7 \times$ $5.4 \mathrm{~mm}$, with evidence of coil compaction at the superior left dome (Fig. 4). Given these findings, a flow diversion treatment strategy was chosen as definitive therapy. While in the endovascular suite, the patient received a loading dose of $325 \mathrm{mg}$ aspirin and $30 \mathrm{mg}$ prasugrel. The pseudoaneurysm was re-coiled with 2 Penumbra SMART coils and 1 Axium Prime coil. Afterward, a $3 \mathrm{~mm} \times 12-\mathrm{mm}$ Pipeline Flex embolization device was introduced and placed from the left $\mathrm{P}_{1}$ posterior cerebral artery (PCA) to the BA (Fig. 5). The patient was uneventfully extubated and discharged the following day. Follow-up angiography
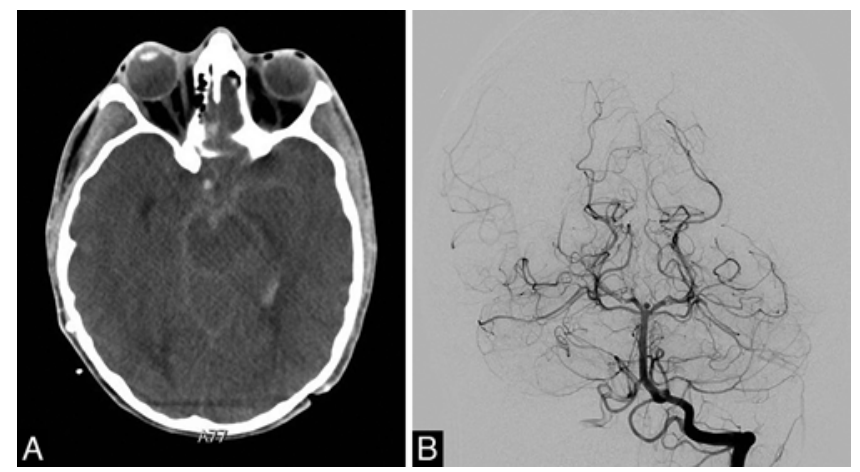

FIG. 2. A: Axial CT angiogram obtained immediately after the aborted ETV demonstrating a 3-mm basilar tip pseudoaneurysm. B: Left vertebral artery angiogram demonstrating a $3-\mathrm{mm}$ basilar tip pseudoaneurysm.

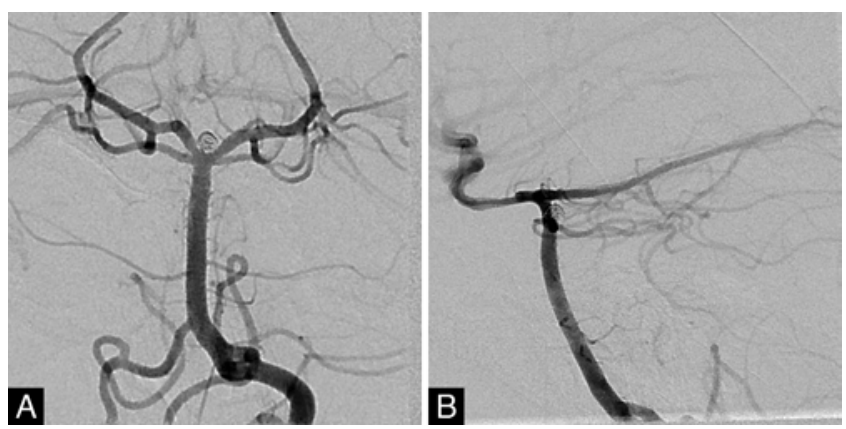

FIG. 3. Anteroposterior (A) and lateral (B) left vertebral artery angiograms demonstrating successful closure of the basilar pseudoaneurysm with 3 extra-soft $1.5 \mathrm{~mm} \times 2-\mathrm{cm}$ Penumbra SMART coils.

conducted 1 month later demonstrated complete obliteration of the aneurysm and complete filling of the right PCA from the right posterior communicating artery (PCOA). These findings were again demonstrated at the 7-month follow-up (Fig. 6), at which time his prasugrel was discontinued.

\section{Discussion}

At long-term follow-up, the success rate of ETV in children has been reported at 50\%-70\%.7,8 Many underlying causes of ETV failure, such as inadequate ostomy diameter, arachnoid scarring, ostomy occlusion by a membrane, and development of a second membrane between the third ventricle and the cisterns have been described.,9,11 Repeat ETV is considered a safe option for ETV failure, with rates of successful treatment ranging from $50 \%$ to $89 \%$.

Intraoperative and postoperative complications of ETV include bleeding; forniceal, hypothalamic, or thalamic injury; meningitis; CSF leak; and new neurological deficit. ${ }^{3,8}$ In a prospectively collected data set from the Hydrocephalus Clinical Research Network, the rates of moderate to severe intraoperative bleeding and major arterial injury were reported to be $6.0 \%$ and $0.3 \%$, respectively. ${ }^{8}$ Basilar artery rupture was identified in $5(0.21 \%)$ of 2344 cases in a large, heterogeneous review. ${ }^{3}$ Although data regarding specific complications in rETV are sparse, no cases of bleeding or aneurysm development in rETV have been reported. ${ }^{11}$

To our knowledge, 3 cases of BA aneurysms have been reported as complications of ETV.,10,12 Horowitz et al. re-

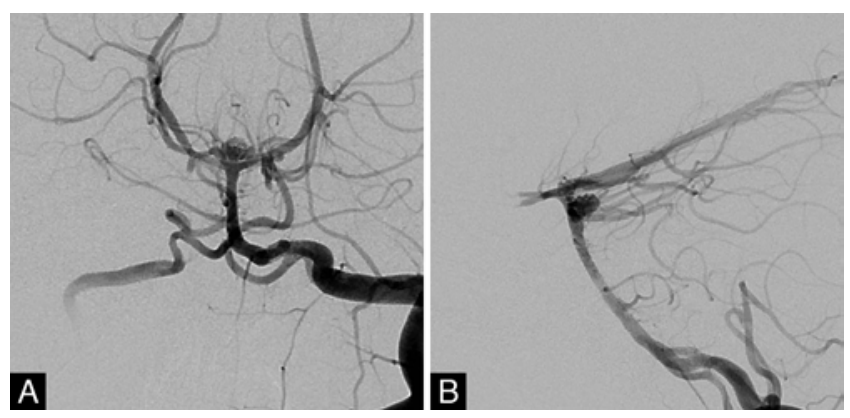

FIG. 4. Anteroposterior (A) and lateral (B) left vertebral angiograms demonstrating gross recurrence and enlargement of aneurysm. 


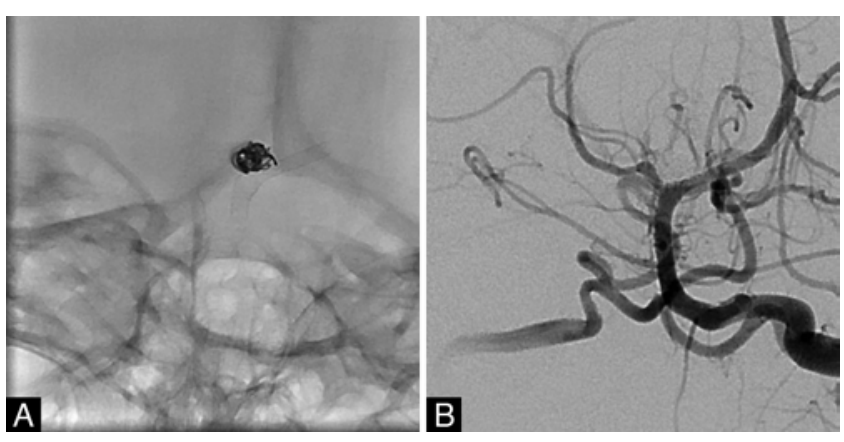

FIG. 5. Left ventricular angiograms demonstrating coiling of the aneurysm (A) and diminished flow through the right PCA (B).

ported the discovery of a 6- to 7-mm saccular aneurysm of the upper BA trunk in a 30-month-old child after third ventricular fenestration with a Bugby wire and low coagulating current. ${ }^{5}$ Because of the presence of a fetal left PCoA and large right PCoA, the authors elected to embolize the aneurysm and the BA directly above and below the aneurysm with Guglielmi detachable coils. Rezende et al. reported the discovery of a 2-mm upper basilar trunk aneurysm in a 4-year-old child following removal of a Fogarty balloon catheter from a fenestration created with a Bugby wire. ${ }^{12}$ The authors elected to treat this aneurysm with coil embolization alone. In both of these cases, the aneurysm was completely obliterated on follow-up imaging. McLaughlin et al. reported the delayed discovery of a 6-mm basilar apex aneurysm in a 3-year-old child 35 days after the original ETV, during which brisk intraoperative bleeding was encountered following the passage of the endoscope through the fenestration to assess CSF flow. ${ }^{10}$ Although the postoperative CT scan demonstrated SAH and IVH, subsequent angiography did not demonstrate an aneurysm. Follow-up interval angiography demonstrated a basilar tip pseudoaneurysm and the patient underwent open surgical clipping. Finally, Abtin et al. reported the development of a 3-mm pseudoaneurysm of the $\mathrm{P}_{1}$ segment of the right PCA following fenestration of the third ventricle floor with an endoscope that resulted in brisk arterial bleeding. ${ }^{1}$ The pseudoaneurysm was subsequently clipped following a pterional craniotomy and, following recurrence 1 month later, clipped again.

Our case highlights an unstable pseudoaneurysm that was treated successfully with coil embolization and subsequent flow diversion. Flow-diverting devices, which offer treatment for aneurysms that may not be amenable to other endovascular interventions, are a relatively novel addition to the armamentarium of aneurysm occlusion devices. The use of a flow-diverting device to treat this pseudoaneurysm in particular demonstrates the reconstructive capabilities of this technology for treating injured vessels. Although the pediatric experience with these devices is limited, no recurrence of an aneurysm treated with flow diversion has been reported in the adult literature. ${ }^{13} \mathrm{Un}$ fortunately these devices currently require the use of dual antiplatelet agents and are used with hesitancy in acutely ruptured lesions. ${ }^{14}$ Furthermore, flow-diverting devices risk development of in-stent thrombosis and loss of branch vessels. This has been specifically noted within the poste-

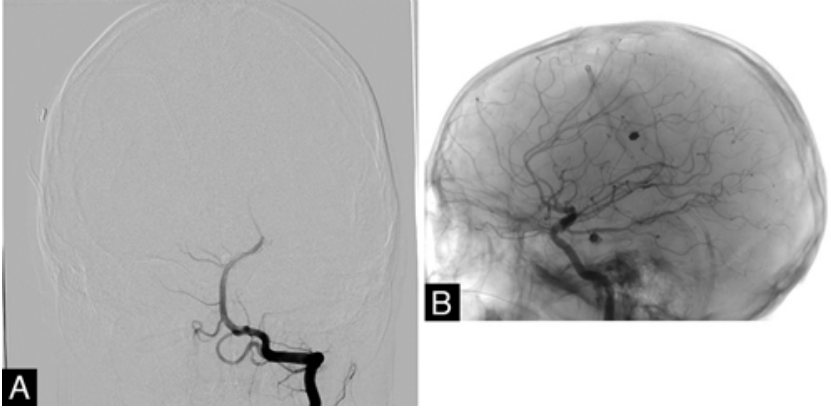

FIG. 6. A: Left vertebral angiogram demonstrating resolution of the lesion with expected loss of flow in the right $P_{1}$ segment of the PCA. B: Right internal carotid artery unsubtracted angiogram demonstrating collateral perfusion of the right PCA filling via the right PCoA.

rior circulation..$^{2,6}$ Yet, as in this case, in circumstances in which treatment options are limited, the risk of conservative management may outweigh the risk of flow diversion.

\section{Conclusions}

Development of a BA aneurysm is a rare but serious consequence of ETV in pediatric patients. We report the case of a 13-year-old patient who developed an aneurysm of the basilar tip following rETV for hydrocephalus. The aneurysm recurred despite initial coil embolization and was definitively treated with flow diversion. To our knowledge, this is the first report of a basilar aneurysm developing during $\mathrm{rETV}$ and of an iatrogenic BA aneurysm being successfully treated with a flow diverter. Further research will be necessary to identify the frequency and etiologies of complications associated with rETV and to determine the durability of treatment of pediatric aneurysms with flow-diverting devices.

\section{References}

1. Abtin K, Thompson BG, Walker ML: Basilar artery perforation as a complication of endoscopic third ventriculostomy. Pediatr Neurosurg 28:35-41, 1998

2. Becske T, Potts MB, Shapiro M, Kallmes DF, Brinjikji W, Saatci I, et al: Pipeline for uncoilable or failed aneurysms: 3-year follow-up results. J Neurosurg 127:81-88, 2016

3. Bouras T, Sgouros S: Complications of endoscopic third ventriculostomy. J Neurosurg Pediatr 7:643-649, 2011

4. Hellwig D, Giordano M, Kappus C: Redo third ventriculostomy. World Neurosurg 79 (2 Suppl):S22.e13-S22.e20, 2013

5. Horowitz M, Albright AL, Jungreis C, Levy EI, Stevenson K: Endovascular management of a basilar artery false aneurysm secondary to endoscopic third ventriculostomy: case report. Neurosurgery 49:1461-1465, 2001

6. Kulcsár Z, Ernemann U, Wetzel SG, Bock A, Goericke S, Panagiotopoulos V, et al: High-profile flow diverter (silk) implantation in the basilar artery: efficacy in the treatment of aneurysms and the role of the perforators. Stroke 41:16901696,2010

7. Kulkarni AV, Drake JM, Mallucci CL, Sgouros S, Roth J, Constantini S: Endoscopic third ventriculostomy in the treatment of childhood hydrocephalus. J Pediatr 155:254-259, 259.e1, 2009

8. Kulkarni AV, Riva-Cambrin J, Holubkov R, Browd SR, Cochrane DD, Drake JM, et al: Endoscopic third ventriculostomy in children: prospective, multicenter results from 
the Hydrocephalus Clinical Research Network. J Neurosurg Pediatr 18:423-429, 2016

9. Marano PJ, Stone SS, Mugamba J, Ssenyonga P, Warf EB, Warf BC: Reopening of an obstructed third ventriculostomy: long-term success and factors affecting outcome in 215 infants. J Neurosurg Pediatr 15:399-405, 2015

10. McLaughlin MR, Wahlig JB, Kaufmann AM, Albright AL: Traumatic basilar aneurysm after endoscopic third ventriculostomy: case report. Neurosurgery 41:1400-1404, 1997

11. Moreira I, Pereira J, Oliveira J, Salvador SF, Vaz R: Endoscopic re-opening of third ventriculostomy: Case series and review of literature. Clin Neurol Neurosurg 145:58-63, 2016

12. Rezende MT, Spelle L, Piotin M, Mounayer C, de Paula Lucas C, Abud DG, et al: Selective endovascular treatment of a traumatic basilar aneurysm after endoscopic third ventriculostomy. Neuroradiology 50:443-446, 2008

13. Vargas SA, Diaz C, Herrera DA, Dublin AB: Intracranial aneurysms in children: the role of stenting and flow-diversion. J Neuroimaging 26:41-45, 2016

14. Walcott BP, Koch MJ, Stapleton CJ, Patel AB: Blood flow diversion as a primary treatment method for ruptured brain aneurysms - concerns, controversy, and future directions. Neurocrit Care 26:465-473, 2017

\section{Disclosures}

Dr. Patel reports being a consultant for Medtronic and Penumbra.

\section{Author Contributions}

Conception and design: Patel, Koch, Grannan, Stapleton, Butler. Acquisition of data: Patel, Sastry, Koch, Butler. Analysis and interpretation of data: Sastry, Koch. Drafting the article: Sastry, Koch, Grannan. Critically revising the article: Koch, Grannan. Reviewed submitted version of manuscript: Sastry, Koch, Stapleton, Butler. Statistical analysis: Grannan, Stapleton. Administrative/technical/material support: Patel.

\section{Correspondence}

Aman B. Patel, Department of Neurosurgery, Massachusetts General Hospital, 15 Parkman St., Boston, MA 02114. email: abpatel@mgh.harvard.edu. 